\title{
Evaluation of Oral Microbial Flora in Saliva of Patients of Oral Submucous Fibrosis
}

\begin{abstract}
Alka Harish Hande ${ }^{1}$, Archana Sonone ${ }^{2}$, Roshni Porwar ${ }^{3}$, Vidya Lohe ${ }^{4}$, Suwarna Dangore ${ }^{5}$, Mrunal Meshram ${ }^{6}$
1Department of Oral Pathology and Microbiology, Sharad Pawar Dental College and Hospital, Datta Meghe Institute of Medical Sciences (Deemed to be University), Sawangi, Wardha, Maharashtra, India. ${ }^{2}$ Department of Oral Pathology and Microbiology, Sharad Pawar Dental College and Hospital, Datta Meghe Institute of Medical Sciences (Deemed to be University), Sawangi, Wardha, Maharashtra, India. ${ }^{3}$ Department of Oral Pathology \& Microbiology, Sharad Pawar Dental College and Hospital, Datta Meghe Institute of Medical Sciences (Deemed to be University), Sawangi, Wardha, Maharashtra, India. ${ }^{4}$ Department of Oral Diagnosis, Medicine and Radiology, Sharad Pawar Dental College and Hospital, Datta Meghe Institute of Medical Sciences (Deemed to be University), Sawangi, Wardha, Maharashtra, India. ${ }^{5}$ Department of Oral Diagnosis, Medicine and Radiology, Sharad Pawar Dental College and Hospital, Datta Meghe Institute of Medical Sciences (Deemed to be University), Sawangi, Wardha,

Maharashtra, India. ${ }^{6}$ Department of Oral Diagnosis, Medicine and Radiology, Sharad Pawar Dental College and Hospital, Datta Meghe Institute of Medical Sciences (Deemed to be University), Sawangi, Wardha, Maharashtra, India.
\end{abstract}

\section{ABSTRACT}

\section{BACKGROUND}

Oral Submucous Fibrosis (OSMF) has a multifactorial origin and the risk factors vary across different parts of the world. Betel nut (areca nut) chewing is the leading cause of OSMF in parts of South East Asia as well as Indian subcontinent. Certain fungal species have been identified in the occurrence of various premalignant lesions including OSMF. Candida in mouth along with the epithelial changes like atrophy, dysplasia, and hypertrophy may predispose individuals to premalignancy. This study was carried out to evaluate the prevalence of microbial flora in saliva of patients of OSMF.

\section{METHODS}

The study included a total of 48 subjects, with 24 clinically diagnosed OSMF patients and a control group of 24 healthy age sex matched patients. Demographic data and salivary samples were collected from both the groups. Swabs taken were subjected for Gram staining and Sabouraud's Dextrose Agar. The positively cultured Candida samples were further analysed by using germ tube test for determining different Candida species.

\section{RESULTS}

In total, $58.3 \%$ of the OSMF patients and $29.2 \%$ of the control patients yielded candida organisms on culture. The difference between the two groups was statistically significant $\left(x^{2}=4.148, p=0.042\right)$. Final results of the study indicate that presence of Candida is significantly higher in OSMF patients than healthy subjects. Thus, results of the present study confirm the phenomenon of high prevalence of oral colonization by yeast in OSMF patients than healthy subjects.

\section{CONCLUSIONS}

In OSMF, there is an altered microbiome which may be used as an indicator for malignant transformation of OSMF. The present study shows higher incidence and intensity of candida in OSMF patients when compared to healthy individuals. Hence, it is mandatory to maintain proper oral hygiene status in OSMF patients to prevent further complications of candidiasis.

\section{KEY WORDS}

Candida albicans, Oral Submucous Fibrosis, Oral Cancer
Corresponding Author: Dr. Alka Harish Hande, Department of Oral Pathology and Microbiology, Sharad Pawar Dental College and Hospital, Datta Meghe Institute of Medical Sciences (Deemed to be University), Sawangi, Wardha-442001, Maharashtra, India. E-mail: alkahande11@gmail.com

DOI: 10.14260/jemds/2020/93

Financial or Other Competing Interests: None.

How to Cite This Article:

Hande AH, Sonone A, Porwar R, et al. Evaluation of oral microbial flora in saliva of patients of oral submucous fibrosis. J. Evolution Med. Dent. Sci. 2020;9(07):409412, DOI: $10.14260 /$ jemds/2020/93

Submission 27-11-2019,

Peer Review 22-01-2020

Acceptance 29-01-2020,

Published 17-02-2020.

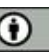




\section{BACKGROUND}

Oral Submucous Fibrosis (OSMF), first described in the early 1950 s is predominantly seen in the people of Asian descent. ${ }^{1}$ Pindborg defined OSMF as "an insidious, chronic disease affecting any part of the oral cavity and sometimes the pharynx. Although occasionally preceded by and/or associated with vesicle formation, it is always associated with juxta epithelial inflammatory reaction followed by fibroelastic change of the lamina propria, with epithelial atrophy leading to stiffness of the oral mucosa and causing trismus and inability to eat". ${ }^{2,3}$ In $600 \mathrm{BC}$, an ancient Indian doctor named Sushruta, described a condition called Vidari, which resembled OSMF and had features of pain on taking food, narrowing of mouth etc. In modern literature OSMF was first described in 1952 in 5 Indian women from Kenya by Schwartz which he named as "atrophia idiopathica mucosar oris". Later, it was termed as OSMF. In India the disease was first described by Joshi in 1953 for the first time and he thereby named it as Submucous fibrosis of palate and pillars. Clinically OSMF presents with reduced mouth opening, burning sensations, blanching and reduced salivary flow rate as well as difficulty in mastication, phonation and deglutition. Histologically, it is associated with juxta epithelial inflammatory cell reaction, followed by fibroelastic changes in lamina propria in turn leading to the stiffness of oral mucosa. 4,5

OSMF has a multifactorial origin and the risk factors vary across different parts of the world. Betel nut (areca nut) chewing is the leading cause of OSMF in the parts of South East Asian as well as Indian Subcontinent. ${ }^{6,7}$ Polyphenols including tannins and various alkaloids are the major chemical components of betel nut.7,8 The fourth most commonly used addictive substance in the world after tobacco, alcohol and caffeine is betel nut. Their prevalence amongst the youths is highest in India. ${ }^{9}$ The upsurge in the popularity and increased uptake of this habit by young people is due to easy access, affordable price, and marketing strategy. In India, the areca nut is used in various combinations; pan masala or kharra (powdered betel nut with tobacco and flavouring agents), betel quid (betel nut, slaked lime, and betel leaf) with or without tobacco, and raw betel nut (flakes or granules). ${ }^{6}$ Thus, betel nut has been referred to as Group 1 human carcinogen. ${ }^{10,11}$ It is a high risk potentially malignant disease and about 7 to $12 \%$ cases of OSMF reports malignant transformation. ${ }^{1,11}$ Earlier this condition was thought to prevail in countries like India, Bangladesh, and Pakistan but now because of higher immigration rates, even reported in western countries, in fact where Indians have settled these conditions are found to be more prevalent over others.

Over 300 microflorae inhabit the oral cavity of normal healthy individual which play an important role in maintaining homeostasis, protection against any pathogenic species. ${ }^{7}$ Certain fungal species have been identified in the occurrence of various premalignant lesions including OSMF. Candida species are the commensals found on the mucosal surface of body and are capable of causing the disease whenever they find the conditions to be favourable. The ability of Candida to colonize, damage the host tissues depends on the imbalance between virulence and host defense. Candida in mouth along with the epithelial changes like atrophy, dysplasia, and hypertrophy may predispose individuals to premalignancy. The most predominant species isolated in premalignancy is
Candida albicans. ${ }^{12,13}$ Candida infection can lead to malignant transformation through the release of nitrosamine compounds, but this process has not been confirmed. Taking these factors into consideration, we have designed this study with the aim to determine the incidence and presence of Candida species in oral cavity of healthy and diseased individuals specially OSMF.

\section{METHODS}

This is a case-control study conducted among 48 participants of which 24 were clinically diagnosed OSMF (cases) and 24 healthy age and sex matched controls. All patients reported to the OPD of Department of Oral Diagnosis, Medicine, Radiology at Sharad Pawar Dental College, Wardha were selected for the study. Patients who were using areca nut containing products, tobacco in various forms and where clinically exhibiting signs and symptoms of OSMF were included in the study. Patients with systemic illnesses like diabetes, history of any antibiotic or steroid usage were excluded from the study.

Approval from the ethical committee was taken before the conduction of research. After explaining the intended procedure, an informed consent was signed from all the patients. Detailed clinical history was recorded from all the patients and clinical staging of OSMF was noted based on the criteria by Ranganthan et al. ${ }^{14}$ The criteria includes the parameters of normal mouth opening (mo), tongue protrusion (tp) and cheek flexibility (cf). Subjects from both the groups were asked to rinse the mouth based on the oral rinse technique described by Samaranayak et al. ${ }^{15}$ Sample was obtained with the help of sterile cotton swab from the site of lesion in case of diseased patients and from normal mucosa in case of control groups. Swabs were cultured on Sabouraud dextrose agar (SDA) using streak culture method. All the plates were then placed in incubator at 370 Celsius for 24-48 hr for the growth of Candida. Gram Staining was further performed on each specimen. The positively cultured Candida samples were further analysed by Germ tube test for determining the different species of Candida (albicans and non-albicans).

\section{Germ Tube Test}

A small inoculum from an isolated Candida colony was picked up and suspended in test tube that contained normal human serum (0.3-0.5 ml). Further mixture was incubated at 42 degree Celsius for $2 \mathrm{hr}$. A drop of this obtained mixture was placed on a clean glass slide and was covered by a cover slip. This was then further examined under microscope. Growths seen were creamy, smooth pasty colonies.

\section{Statistical Analysis}

All the obtained information was analyzed by using SPSS Software (version 17). Student t test was used to find out the statistical significance. We got the $p$ value of 0.05 which was considered statistically significant.

\section{RESULTS}

In this study, 48 participants were equally divided into two groups- Study and Control groups. The participant's age 
ranged from 19-59 years. A total of 24 OSMF patients comprising of 17 males (70.83\%) and 7 females (29.16\%) with an age range of 19-59 years (Table-1). When gender wise data for occurrence of OSMF was analysed, these lesions were found to be less prevalent in females as compared to males. $19.75 \mathrm{~mm}$ was the average mouth opening of OSMF patients.

\section{Healthy Subjects (HS)}

The HS consists of apparently healthy individuals. HS were matched according to age and sex. HS had 10 males (41.66\%) and 14 females (58.33\%). Any of the patients do not have oral infections or received any antifungal agents. When age wise data was analysed, these lesions were predominately present in $2^{\text {nd }}$ and $4^{\text {th }}$ decade of life. When stage wise data for occurrence of OSMF was analysed, these lesions were strikingly more prevalent in stage 2 .

In total, $58.3 \%$ of the OSMF patients and $29.2 \%$ of the control patients yielded candida organisms on culture. The difference between the two groups was statistically significant $\left(x^{2}=4.148, p=0.042\right)($ table-2). Thus, final results of the study indicate that presence of Candida is significantly higher in OSMF patients than HS. Thus, the results of the present study confirm the phenomenon of high prevalence of oral colonization by yeast in OSMF patients than HS.

\begin{tabular}{|c|c|c|c|c|}
\hline Gender & OSMF N=24 & Control N=24 & $\mathbf{X}^{\mathbf{2}}$ & p-Value \\
\hline Male & $17(70.83 \%)$ & $10(41.66 \%)$ & \multirow{2}{*}{0.000} & 1.000 \\
\hline Female & $07(29.16 \%)$ & $14(58.33 \%)$ & & \\
\cline { 1 - 1 } Table 1. Gender Wise Distribution of Subjects in OSMF \\
Group and Control Group \\
\hline
\end{tabular}

\begin{tabular}{|c|c|c|c|c|}
\hline C. albicans & OSMF N=24 & Control N=24 & $\mathrm{X}^{2}$ & p-Value \\
\hline Present & $14(58.3 \%)$ & $7(29.2 \%)$ & \multirow{2}{*}{4.148} & \multirow{2}{*}{$0.042^{*}$} \\
\hline Absent & $10(41.7 \%)$ & $17(70.8 \%)$ & & \\
\cline { 1 - 2 } Table 2. Distribution of Candida Species in OSMF Group \\
and Control Group \\
\hline \multicolumn{5}{|c}{${ }^{*}<0.05 ;$ Significant }
\end{tabular}

\section{DISCUSSION}

Premalignant disorders are indicators of risk for likely future malignancies. It is now very well known that even a clinically normal appearing mucosa in patient having a precancerous lesion may have dysplasia on contralateral anatomic sites or other mucosal sites suggestive of a pathway for malignant transformation. The pathogenesis for these lesions is multifactorial. OSMF is the most common potentially malignant disorder. Epidemiologic studies that included caseseries reports, large cross-sectional surveys, and case-control studies have identified areca nut as the major etiologic agents. Areca nut chewers show alteration of oral bacterial micro biome. Prolonged use can lead to alteration of oral micro biome including reduced level of commensal bacteria which is thereby critical to maintain homeostasis. The synergistic effects of these factors have high carcinogenic potency. Tissueculture studies involving human fibroblasts, areca nut extracts and areca nut alkaloids supported this etiologic hypothesis by showing fibroblastic proliferation and increased collagen. ${ }^{7}$ The other low risk factors like syphilis, genetic susceptibility, hormonal disturbances, UV radiation etc are also associated with these lesions. They too have synergistic role with high risk factors in carcinogenesis. ${ }^{12,13}$
Healthy individuals have $3-47 \%$ Candida as a commensal inhabitant of oral mucosa.4,15 It plays major role in oral carcinogenesis through nitrosamines, acetaldehyde, and oligosaccharides production. The most common organism in the oral cavity which has potential to infect any tissue within the body under immunocompromised condition is Candida albicans whereas Non albicans are rarely seen.12,16,17 Candida albicans is the one which is highly infectious amongst Candida species. Non albicans usually lacks the virulence factor totally or partially. Recently, non-candida albicans are isolated from oral mucosa in various oral lesions predominantly premalignant disorders. Absolute role of non albicans in neoplastic transformation is still obscure. ${ }^{18}$

Results of our study suggests that when gender wise analysis of data was done with respect to OSMF, it was found that prevalence of disease was more in males as compared to females. Thus, a definite predilection in males has been observed. This is in accordance with previous studies. ${ }^{19,20}$ Maximum incidence was recorded in second to fourth decades of life. These findings were in accordance with Wahi PN et al. ${ }^{21}$ Candida albicans plays an important role in the development of oral cancer by means of endogenous nitrosamine, oligosaccharides and lectin like component production. ${ }^{12}$ Our results were in accordance with that of Choudhary $\mathrm{M}^{22}$ who suggested that Candidal carriage is significantly higher in OSMF than in HS. Our results also confirmed the findings of Ariyawardana et al, who suggested high candidal carriage in OSMF patients. ${ }^{23}$ On the contrary, Riechart et al. reported that there was no significant difference in Candida carriage between both groups. ${ }^{24}$ Presence of Candida not seen in grade 4 OSMF patients, the reason might be because of small sample size as this study was done on random no of patients. In the present study highest amount of Candida was seen in grade 2 OSMF patients. The presence and association of Candida in our study was ruled out by various investigations using Gram stain, Germ tube test and Sabouraud's agar medium. The present study has thus revealed that Candida carriage was significantly higher in OSMF patients than in healthy individuals. 58.3\% OSMF patients and 29.2\% Control patients yielded Candida organisms on culture.

\section{CONCLUSIONS}

The present study shows higher incidence and intensity of candidiasis in oral submucous fibrosis patients when compared to healthy individuals. Hence, it is mandatory to maintain proper oral hygiene status in oral submucous fibrosis patients so as to prevent further complications of candidiasis (role in carcinogenesis).

\section{REFERENCES}

[1] Tilakaratne WM, Klinikowski MF, Saku T, et al. Oral submucous fibrosis: review on aetiology and pathogenesis. Oral Oncol 2006;42(6):561-8. 
[2] Tekade SA, Chaudhary MS, Tekade SS, et al. Early stage oral submucous fibrosis is characterized by increased vascularity as opposed to advanced stages. Journal of Clinical and Diagnostic Research 2017;11(5):ZC92-ZC6.

[3] Rajendran R. Oral submucous fibrosis: etiology, pathogenesis and future research. Bulletin of the World Health Organization 1994;72(6):985-96.

[4] Gupta B, Chandra S, Raj V, et al. Comparison of salivary flow and Candidal carriage in patients with oral submucous fibrosis. J Oral Maxillofac Pathol 2015;19(2):158-63.

[5] Pindborg JJ, Singh B. Formation of vesicles in oral submucous fibrosis. Acta Pathol Microbiol Scand 1964;62(4):562-6.

[6] Hande AH, Chaudhary MS, Gadbail AR, et al. Role of hypoxia in malignant transformation of oral submucous fibrosis. Journal of Datta Meghe Institute of Medical Sciences University 2018;13(1):38-43.

[7] Hernandez BY, Zhu X, Goodman MT, et al. Betel nut chewing, oral premalignant lesions and the oral microbiome. PLoS One 2017;12(2):e0172196.

[8] Papke RL, Horenstein NA, Stokes C. Nicotinic activity of arecoline, the psychoactive element of "Betel Nuts", suggests a basis for habitual use and anti-inflammatory activity. PLoS One 2015;10(10):e0140907.

[9] Gupta PC, Ray CS. Epidemiology of betel quid usage. Annals of the Academy of Medicine, Singapore 2004;33(Suppl 4):31-6.

[10] Shah G, Chaturvedi P, Vaishampayan S. Arecanut as an emerging etiology of oral cancers in India. Indian J Med Paediatr Oncol 2012;33(2):71-9.

[11] Hande AH, Chaudhary MS, Gawande MN, et al. Oral submucous fibrosis: an enigmatic morpho-insight. J Cancer Res Ther 2019;15(3):463-9.

[12] More C, Peter R, Nishma G, et al. Association of Candida species with oral submucous fibrosis and oral leukoplakia; a case control study. Ann Clin \& Lab Res 2018;6(3):248.

[13] Anila K, Hallikeri K, Shubhada C, et al. Comparative study of Candida in oral submucous fibrosis and healthy individuals. The Revista Odonto 2011;26(1):71-6.
[14] Ranganathan K, Devi U, Elizabeth J, et al. Mouth opening, cheek flexibility and tongue protrusion parameters of 800 normal patients in Chennai, South India. A base line study to enable assessment of alterations in oral submucous fibrosis. J Indian Dental Association 2001;72:78-80.

[15] Samaranayake LP, MacFarlane TW, Lamey P-J, et al. A comparison of oral rinse and imprint sampling techniques for the detection of yeast, coliform and Staphylococcus aureus carriage in the oral cavity. J Oral Pathol 1986;15(7):386-8.

[16] Nada V, Marija BB, Vuckovic D, et al. Presence of Candida albicans in potentially malignant oral mucosal lesions. Arch Oncol 2004;12(1):51-4.

[17] Sahay A. Occurrence of Candida albicans in oral leukoplakia: a clinical and histological evaluation. Ind J of Dent Edu 2012;5(3):137-45.

[18] Birman EG, Kignel S, Da Silveira FR, et al. Candida albicans: frequency and characterization in oral cancer (Stage I) from smokers and drinkers. Rev Iberoam Micol 1997;14(3):101-3.

[19] Hazarey VK, Erlewad DM, Mundhe KA, et al. Oral submucous fibrosis: study of 1000 cases from central India. J Oral Pathol Med 2007;36(1):12-7.

[20] Angadi PV, Rao SS. Areca nut in pathogenesis of oral submucous fibrosis: revisited. Springer- Oral Maxillofac Surg 2011;15(1):1-9.

[21] Wahi PN, Kapur VL, Luthra UK, et al. Submucous fibrosis of the oral cavity. 1. Clinical features. Bull World Health Organ 1966;35(5):789-92.

[22] Chaudhary M, Chandak T, Gawande M, et al. Candidal carriage in oral submucous fibrosis patients - a case control study. J Datta Meghe Inst Med Sci Univ 2012;7(1):5-9.

[23] Ariyawardana A, Panagoda GJ, Fernando HN, et al. Oral submucous fibrosis and oral yeast carriage - a case control study in Sri Lankan patients. Mycoses 2007;50(2):116-20.

[24] Reichart PA, Schmidtberg W, Samaranayake LP, et al. Betel quid-associated oral lesions and oral Candida species in a female Cambodian cohort. J Oral Patho Med 2002;31(8):468-72. 